\section{Sharps injuries in ophthalmic practice}

\author{
A-J Ghauri' ${ }^{1}$ KN Amissah-Arthur ${ }^{2}$, A Rashid ${ }^{3}$, \\ B Mushtaq ${ }^{1}, \mathrm{M} \mathrm{Nessim}^{1}$ and S Elsherbiny ${ }^{1}$
}

\begin{abstract}
Purpose Accidental sharps injuries are a potential route for transmission of blood-borne infection to healthcare workers. Ophthalmic staff in particular are at risk of sustaining such injuries due to the microsurgical nature of the speciality. Forthcoming European Union legislation aimed at reducing sharps injuries requires the development of risk-based sharps policy. The authors believe that this is the first study to assess the risks of sharps injuries and their management specific to ophthalmic practice within the European Union.

Methods A retrospective review of all reported sharps injuries across three eye units in the UK over a period of 6 years was undertaken. Data were analysed to determine the circumstances surrounding the injury, occupation of the injured person, and whether appropriate actions were taken following incidents.
\end{abstract}

Results A total of 68 sharps injuries were reported over the 6-year period. Nurses sustained $54.4 \%(n=37)$ of needlestick injuries, doctors $39.7 \%(n=27)$, and allied healthcare staff $5.9 \%(n=4)$. In all $51.5 \%(n=35)$ of sharps injuries occurred in the operating theatre, $30.9 \%$ $(n=21)$ in the outpatient clinic, $13.2 \%(n=9)$ on the ophthalmic ward, and $4.4 \%(n=3)$ in unspecified locations. There was a median rate of 1.3 sharps injuries per 1000 surgical procedures per year and a range of 0.4-3.5 per 1000 .

Conclusions This study demonstrates the need to raise awareness of the unique risks of sharps injuries in ophthalmic practice. This is necessary in order to develop specialityspecific policy that promotes strategies to reduce such injuries, enhances the accuracy of reporting of such events, and provides guidance for appropriate management. Eye (2011) 25, 443-448; doi:10.1038/eye.2011.13; published online 18 February 2011

Keywords: sharps injury; occupational exposure; ophthalmology; medico-legal

\section{Introduction}

Occupational sharps injuries are associated with a risk of transmission of blood-borne viruses (BBVs) to health care workers (HCWs). ${ }^{1}$ They are relatively common, with $\sim 40000$ such incidents reported annually among United Kingdom NHS staff. ${ }^{2}$ They are the second most common cause of occupational injury and account for $17 \%$ of reported work-related accidents. $^{3}$

Due to an increased incidence of sharps injuries across all healthcare settings, ${ }^{1}$ an increased prevalence of BBVs in the general community $^{4}$ and a greater number of patients with confirmed BBVs accessing healthcare services, ${ }^{1}$ HCWs are being placed under ever increasing risks of acquiring BBVs. The United Kingdom health protection authority (HPA) ${ }^{1}$ reported that between 2000-2007 most occupational sharps injuries involved nursing professionals (48\%), however, medical professionals carried a disproportionate burden of significant exposures (ie, exposure to a patient confirmed to be positive for a BBV) and exposures in this group increased by $78 \%$. Between 1997 and 2007 there were a total of 14-documented hepatitis C seroconversions. ${ }^{1}$ In two of these cases, the HCW involved was wearing gloves at the time of the incident. Throughout the same time period there has been one documented and 23 probable HIV seroconversions. ${ }^{1}$ (Incidents defined as 'probable' relate to HCWs in whom the only significant risk factor for HIV seroconversion was an occupational exposure, but who did not have a negative baseline HIV test at time of exposure.) The consequences of a sharps injury reach far beyond the immediate risk of BBV acquisition as such events are highly stressful and have the potential to affect an individual's

The operating theatre environment is the second most common location in which sharps injuries occur and was responsible for $17 \%$ of the high risk sharps injuries reported to the HPA in 2007. ${ }^{1}$ The risks for sharps injuries arise because surgical procedures are often blood career, family, and patients.
${ }^{1}$ Birmingham and Midland Eye Centre, Birmingham, West Midlands, UK

${ }^{2}$ University Hospitals Birmingham, Selly Oak Hospital, Birmingham, West Midlands, UK

${ }^{3}$ Wolverhampton and Midland Counties Eye Infirmary, Birmingham West Midlands, UK

Correspondence: A-J Ghauri, Birmingham and Midland Eye Centre, City Hospital, Dudley Road, Birmingham, West Midlands B18 7QH, UK.

Tel: + $44(0) 1215076849$; Fax: + 44 (0)121 5076853 E-mail:ajghauri@doctors. org.uk

Received: 15 November 2010

Accepted in revised form: 13 December 2010 Published online: 18 February 2011

This work has been presented at the Royal College of Ophthalmologists Annual Congress 2009. 
intensive, involve manipulation of sharp instruments, and require highly coordinated interactions between members of the surgical team. ${ }^{5}$

The unique microsurgical nature of ophthalmic practice presents further risks for sustaining sharps injuries. ${ }^{6}$ There is an increased likelihood of instrument mishandling due to the use of fine instruments and sutures. Surgeons also often operate under magnification and with minimal background lighting, which can further increase the risk of instrument mishandling outside of a narrow surgical field. Mansour et $a l^{7}$ reported that these special circumstances contributed to the finding that when compared with other hospital specialities, ophthalmologists were at the greatest risk for sustaining a sharps injury.

The European Union (EU) has recently ratified directive 2010/32/EU on the prevention of sharps injuries in hospital and healthcare settings. Member countries must pass this into law by 11 May $2013 .^{8}$ The NHS has published guidelines to ensure healthcare organisations are ready and compliant once the United Kingdom legislation is passed. 3,9 They are required to develop sharps injury policy based on individual risk, promote strategies to reduce injuries, and ensure robust mechanisms are in place for the investigation, management, and reporting of such injuries. In 2010/2011, the United Kingdom government's health and safety executive will be undertaking an inspection of sharps injury policy across NHS trusts on the British mainland. ${ }^{10}$

To our knowledge, there are no published studies addressing the issue of sharps injuries specific to ophthalmic practice outside of the United States of America. Moreover, the most recent studies addressing sharps injuries in ophthalmology are over 20 years old. ${ }^{6,7}$

The aims of this study were therefore to:

- Establish the incidence of reported sharps injuries in ophthalmic practice in three United Kingdom institutions.

- Establish the risk factors for sustaining a sharps injury in the ophthalmic settings.

- Assess how well the sharps injury safety policy is implemented.

\section{Materials and methods}

The term 'sharps injury' is defined as a percutaneous exposure, where the skin has been broken by a needle or any other sharp object contaminated by blood or other bodily fluid. It is often used interchangeably with the term 'needlestick injury'.

Data on all sharps injuries over a 6-year period were obtained from critical incident forms and a review of the sharps injury database at three ophthalmology units in the West Midlands, UK. These three units were chosen because they were the hospitals where the authors worked. Hospital protocol ${ }^{11}$ requires all sharps injuries or near misses to be reported via the critical incident form. Data were collected on individual incidents, including the device that caused the injury, the mechanism of injury, the individual affected, and the post exposure management performed. All reported near misses have also been included in the study. The data were analysed and all statistics were performed using Microsoft Excel 2008 version 12 on Macintosh. (Redmond, WA, USA).

\section{Results}

A total of 68 sharps injuries were reported from the three ophthalmic units over the 6-year period (Table 1 ). Nurses sustained $54.4 \%(n=37)$ of injuries, $39.7 \%(n=27)$ were sustained by doctors, and $5.9 \%(n=4)$ by allied healthcare staff. The majority of sharps injuries occurred in the operating theatre. $51.5 \%(n=35)$. In the outpatient clinic, $30.9 \%(n=21)$ injuries were sustained, $13.2 \%$ $(n=9)$ of sharps injuries occurred on the ophthalmic ward and $4.4 \%(n=3)$ had an unspecified location.

There was a varying rate of incidents per 1000 surgical procedures per year over the 6-year period (Table 2). There was a median rate of 1.3 sharps injuries per 1000 surgical procedures per year and a range of 0.4-3.5 per 1000 .

Table 1 Distribution of sharps injuries by occupation of injured $\mathrm{HCW}$, context of injury, location of injury and appropriateness of action taken by HCW (2004-2009)

\begin{tabular}{|c|c|c|c|c|c|}
\hline Occupation & $\begin{array}{l}\text { Unit } A \\
\text { number }\end{array}$ & $\begin{array}{l}\text { Unit B } \\
\text { number }\end{array}$ & $\begin{array}{l}\text { Unit C } \\
\text { number }\end{array}$ & Total & $\%$ \\
\hline Doctors & 5 & 15 & 7 & 27 & 39.7 \\
\hline Nurses & 4 & 26 & 7 & 37 & 54.4 \\
\hline Other staff & 0 & 2 & 2 & 4 & 5.9 \\
\hline \multicolumn{6}{|l|}{ Context } \\
\hline Procedural & 4 & 15 & 8 & 27 & 39.7 \\
\hline Passing instrument & 2 & 1 & 2 & 5 & 7.4 \\
\hline Disposal and cleaning & 2 & 23 & 6 & 31 & 45.6 \\
\hline Unspecified & 1 & 4 & 0 & 5 & 7.4 \\
\hline \multicolumn{6}{|l|}{ Location } \\
\hline Theatre & 3 & 26 & 6 & 35 & 51.5 \\
\hline OPD & 5 & 9 & 7 & 21 & 30.9 \\
\hline Ward & 1 & 6 & 2 & 9 & 13.2 \\
\hline Sterile services & 0 & 0 & 1 & 1 & 1.5 \\
\hline Not specified & 0 & 2 & 0 & 2 & 2.9 \\
\hline \multicolumn{6}{|l|}{ Action } \\
\hline Correct & 4 & 29 & 10 & 43 & 63.2 \\
\hline Incorrect & 1 & 8 & 1 & 10 & 14.7 \\
\hline Not specified & 3 & 4 & 4 & 11 & 16.2 \\
\hline Near miss & 1 & 2 & 1 & 4 & 5.9 \\
\hline
\end{tabular}


The rates varied from unit to unit with the smaller ophthalmology unit incurring more sharps injuries per 1000 procedures per year (median; 2.65 per 1000) than the two larger hospitals (medians; 1 and 1.15 per 1000).

The correct post-exposure first aid actions were documented in $63.2 \%(n=43)$ of injuries. This was defined according to national guidelines as immediately 'encouraging bleeding of the wound, which should be washed with soap or chlorhexidine and water, but not scrubbed or sucked'. ${ }^{3}$ (Figure 1) The incorrect action was taken by $14.7 \%(n=10)$ of injured healthcare workers, $16.2 \%(n=11)$ of HCWs did not specify the action they took post exposure. There were four near misses; these did not require the same reporting requirements as actual sharps injuries.

The majority of injuries occurred during the disposal of sharps $(45.6 \%)$. This included six injuries to individuals who were not the original users of the instruments as a result of sharps inappropriately discarded by others. Of two notable incidents, one included a surgeon stepping on a needle that was lost in an earlier operating list and in another, a domestic staff member sustained an injury from a used syringe that had been inappropriately discarded in a thin-walled refuse sac. There were also four near misses where sharp tipped instruments or needles had been inappropriately discarded; two of these had been discarded in a clinical waste bin. A near miss also occurred when a medical sharp was found in a theatre scrub garment that had been freshly returned from outsourced laundry services.

Table 2 Rates of sharps injuries per 1000 surgical procedures a year (2004-2009)

\begin{tabular}{lccc}
\hline Hospital & Year & Number of NSI & Rate per 1000 \\
\hline Unit A & 2007 & 6 & 3.5 \\
\multirow{3}{*}{ Unit B } & 2008 & 3 & 1.8 \\
& 2004 & 3 & 0.4 \\
& 2005 & 9 & 1.3 \\
& 2006 & 11 & 1.6 \\
& 2007 & 10 & 1.4 \\
Unit C & 2008 & 3 & 0.4 \\
& 2009 & 7 & 1 \\
& 2008 & 4 & 0.8 \\
Median & 2009 & 5 & 1.4 \\
\hline
\end{tabular}

There were 19 (30\%) injuries attributable to hollow-bore instruments, 13 among nursing staff, five among doctors, and one sharps injury to a domestic cleaning staff. The source patient was known in all of these cases.

\section{Discussion}

This study confirms the small, but very real risk of sharps injuries in ophthalmic practice. Nursing staff (54.4\%) was most at risk of sustaining a sharps injury followed by doctors $(39.7 \%)$. These findings are consistent with data from the $\mathrm{HPA}^{1}$ where nursing staff bear the greatest burden of sharps injuries across all healthcare settings. When examining all three units, the greatest incidence of sharps injuries occurred in the operating theatre setting $(51.5 \%)$. This is in contrast to findings by the HPA ${ }^{1}$ that for all healthcare settings in England, the operating theatre environment is the second most common location for sustaining a sharps injury (17\%) with the greatest incidence of sharps injuries $(36 \%)$ occurring on the in-patient wards. In this study, the risk of sustaining a sharps injury on the in-patient wards was only $13.2 \%$. The unique distribution of sharps injuries can be explained by the findings that ophthalmology has a very high surgical throughput, but very low inpatient load. ${ }^{12}$ In 2004-2010 a total of 2615809 ophthalmic surgical procedures were performed in the UK. ${ }^{12}$ This included 2349284 day case procedures, which was the highest among all hospital specialities. ${ }^{12}$ Ophthalmology also had the lowest inpatient load among the main surgical specialities with a mean inpatient stay of 1.3 days. ${ }^{12}$

It is also important to be aware of the potential for sharps injuries to occur in locations outside of the operating theatre environment as a high incidence of sharps injuries occurred in the outpatient setting (30.9\%). This can be attributed to the large number of office-based invasive procedures performed in the ophthalmic clinic. Thus when performing office-based procedures, one must remain vigilant and exercise the same precautions with sharp instruments as taken in the operating theatre environment.

In this study, the majority of injuries occurred during the disposal of sharps (45.6\%). This included six injuries to individuals who were not the original users of the

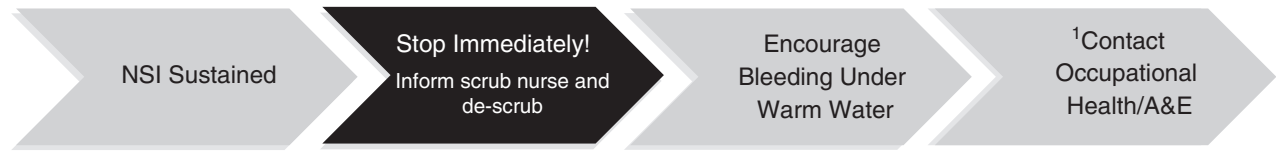

${ }^{1}$ All subsequent decisions regarding post-exposure prophylaxis are dependent on establishing an individual level of risk and on each hospitals protocol.

Figure 1 A flowchart of universal first aid instructions following sharps injury. 
instruments. Such injuries are particularly serious as it is very difficult to identify the source patient. This can lead to a prolonged course of post exposure prophylaxis and months of anxiety for the individual concerned until the absence of seroconversion can be confirmed. Such injuries are not restricted to original users of the instruments as they have the potential to affect any individual working in a clinical area or coming into contact with clinical waste. ${ }^{13}$ These can be entirely prevented by following universal procedures for correct handling and disposal of clinical waste. In particular, HCWs must ensure that sharps are only disposed of in the correct punctureproof receptacles, which must not be over-filled.

The risk of transmission of BBV is higher when hollow-bore instruments such as intravenous cannulae and hypodermic needles are used. ${ }^{14}$ Greene et $a l^{14}$ found a risk of sharps injury in anaesthetists with hollow-bore instruments to be $87 \%$, this was lower in this study at $30 \%$. This discrepancy is probably due to the increased phlebotomy-based procedures performed by anaesthetists when compared with ophthalmologists.

The bulk of sharps injuries in the operating theatre occurred as the device was being used or passed between HCWs; these included the majority of sharps injuries sustained by doctors (63\%). These observations are already recognised in general surgery, ${ }^{5}$ which has seen the development of various techniques for reducing the use of sharp instruments during surgery and strategies to minimise the risk of sharps injuries during the passing of sharp instruments.

The technique of hands-free passing of sharp instruments, using a neutral zone rather than hand-tohand passing is an established strategy for minimising sharps injuries. This method has been shown to reduce such injuries by $35-59 \%$ in certain types of operations ${ }^{15,16}$ and can be applied to most ophthalmic procedures including the requirement for the surgeon to personally mount and dispose of the suture needle. Hands-free passing is limited by the requirement for the surgeon to look away from the surgical field, which may not be practical during critical aspects of the procedure where constant visualisation of the operative field is required.

A variety of safety-scalpel and keratome devices and self-blunting intravenous cannulae are currently available commercially, which use a sheathed or retractable mechanism to guard the blade/needle when it is not in use. They not only minimise risk when exchanging instruments during a surgical procedure, but also reduce the risk of sustaining a sharps injury when disposing of instruments after the procedure is complete. The widespread uptake of these devices is limited by their cost. Some of the devices have usability issues that also detract from their incorporation into standard ophthalmic practice. The authors were unable to find data evaluating the effectiveness of such mechanical devices in reducing sharps injuries.

General surgery has demonstrated the feasibility of reducing the use of sharp instruments during certain surgical procedures. ${ }^{17}$ Replacing sharp suture needles with blunt ones for suturing muscle and fascia has been highly effective in reducing sharps injuries, ${ }^{18}$ however, outside of a small number of oculoplastic procedures, blunt suture needles have a limited role in ophthalmology.

Tissue adhesives such as cyanoacrylate and fibrin glues have a more significant role in ophthalmology and provide an alternative to conventional sutures across a wide range of surgical procedures. ${ }^{19-21}$ Alternatives to steel surgical scalpel blades for oculoplastic procedures include radio-frequency scalpels, $\mathrm{CO}_{2}$ laser, and argon laser. These instruments provide the benefits of simultaneously coagulating tissues resulting in a bloodless field and minimising the risk of splash injuries. Excimer laser offers a similar alternative to sharp instruments in anterior segment surgery. Ophthalmic surgery is subject to constant innovations and an ever-increasing application of novel techniques that reduce the use of sharp instruments. Adopting any new technique requires a period of learning and adjustment. Novel methods must also be evaluated in light of risks, benefits, and costs.

When attempting to evaluate post-exposure management, it was found that the correct first-aid actions (Figure 1) were only adhered to in $63.2 \%$ of incidents. Although this study is small and retrospective, there is no reason to believe the findings are not representative of a wider problem. Poor adherence to sharps injury policy has also been reported by Thomas and Murray ${ }^{22}$ who found only $9 \%$ of surgeons in a NHS hospital setting correctly followed sharps injury policy following an injury and included $21 \%$ who simply ignored the injury and carried on operating. This neglect of sharps injury policy may result from multiple factors, for example failure to appreciate the seriousness of such an injury, ${ }^{23}$ the pressure to carry on with an operating list or a perceived fear of consequences to one's health and career following a sharps injury. These concerns as well as an ignorance of sharps injury guidance may result in a low adherence rate of sharps injury policy. ${ }^{22}$

The under-reporting of sharps injuries is also well established ${ }^{24-26}$ and the major factors limiting the accuracy of a study such as this are its retrospective nature and the reliance on self-reporting of such injuries. Performing a prospective study would reduce some of these limitations and would have a further advantage of actively raising awareness of sharps injuries thereby improving the reporting rate and the correct post exposure management of such injuries. 
This study also highlights the inconsistencies in the sharps injury databases at the various units. In one unit it was suspected that due to a move from one site to another some of the files pertaining to sharps injuries had been stored in the incorrect location. In another unit due to a change in the method of reporting critical incidents there appeared to be a 'fallow' period in terms of rates of sharps injuries.

The authors believe that education and training is at the core of a robust sharps policy. Individuals must be made aware of the potential risks of such injuries to their own selves and others and informed of universal precautions for safe handling and disposal of sharps. Instruments and techniques that reduce the use of sharps should also be proactively encouraged provided their efficacy and safety has been proven. Yet in spite of the best strategies to minimise the risk of sharps injuries, the authors acknowledge that such injuries will inevitably occur. Thus it is necessary to develop and proactively disseminate clear guidance detailing the reporting and management of such injuries. Local eye units should maintain a record of all sharps related incidents as well as send a copy to the appropriate risk manager at the trust. This duplication would improve the analysis of sharps injury rates and safeguard the data.

In view of the unique risks of sharps injuries in ophthalmology, the authors believe it is necessary to develop speciality-specific guidance for the prevention and management of such injuries. This will also enable ophthalmic units to prepare for forthcoming EU regulations, which will make the adherence to sharps policy a legal requirement. ${ }^{8}$ Furthermore, the authors advocate explicitly incorporating sharps awareness into the ophthalmology speciality curriculum so that it can

\section{Summary}

What was known before

- Sharps injuries are a potential route of transmission of blood-borne infection to healthcare workers.

- Among hospital specialties, ophthalmologists are at greatest risk of sustaining a sharps injury.

- Forthcoming European Union legislation will make the development of risk-based sharps policy a legal requirement.

What this study adds

- The majority of sharps injuries occur during the disposal of instruments and most frequently in the operating theatre environment.

- Most injuries can be completely eliminated through adherence to universal precautions for handling and disposal of sharps.

- Ophthalmologists should evaluate methods reducing sharp instrument usage.

- HCWs need to be familiar with local strategies for the management and reporting of sharps injuries. become embedded in the minds and practice of ophthalmic trainees who will take it forward through their career.

\section{Conflict of interest}

The authors declare no conflict of interest.

\section{References}

1 Health Protection Agency. Eye of the needle: 2008 (cited 9 Sep 2010): http://www.hpa.org.uk/Publications/ InfectiousDiseases/BloodBorneInfections/0811 Eyeoftheneedle/.

2 NHS Employers. Prevention of sharps injuries framework agreement. 6 July 2010 (cited 9 Sep 2010): http:/ / www. nhsemployers.org/HealthyWorkplaces/HealthAndSafety_ new/sharpsinjuriesframework/Pages/

ECFrameworkagreementonsharpsinjuries.aspx.

3 NHS Employers. Needlestick injury. (cited 9 Sep 2010): http://www.nhsemployers.org/Aboutus/Publications / Documents?Needlestick\%20injury.pdf.

4 Health Protection Agency. Shooting up: infections among injecting drug users in the United Kingdom 2008. Oct 2009 (cited 12 Sep 2010): http:/ /www.hpa.org.uk/Topics/ InfectiousDiseases/InfectionsAZ/InjectingDrugUsers/ GeneralInformation/idu_ShootingUp/.

5 Jagger J, Berguer R, Phillips EK, Parker G, Gomaa AE. Increase in sharps injuries in surgical settings versus nonsurgical settings after passage of national needlestick legislation. J Am Coll Surg 2010; 210(4): 496-502.

6 Mansour AM. Needlestick injuries in ophthalmology. Ophthalmic Surg 1989; 20(5): 367-369.

7 Mansour AM. Which physicians are at high risk for needlestick injuries? Am J Infect Control 1990; 18(3): 208-210.

8 The European Union. Council directive 2010/32/EU of 10 May 2010 implementing the framework agreement on prevention from sharp injuries in the hospital and healthcare sector concluded by HOSPEEM and EPSU. (cited 9 Sep 2010): http:/ /eur-lex.europa.eu/JOHtml.do? uri $=$ OJ:L:2010:134:SOM:EN:HTML

9 NHS Employers. Health and safety essential guide. 9 Jun 2010 (cited 9 Sep 2010); http:/ /www.nhsemployers. org/HealthyWorkplaces/HealthAndSafety_new/ HealthAndSafetyEssentialGuide/Pages/HealthAnd Safety.aspx.

10 HSE. Needlesticks and sharps inspection campaign 2010/ 2011. (cited 9 Sep 2010): http://www.hse.gov.uk/ healthservices/needlesticks/inspection-campaign.htm.

11 Consultant Occupational Physician. Blood contamination/ needlestick policy. Sep 2008. SWBH NHS Trust: Birmingham.

12 Health and Social Care Information Centre. Hospital episode statistics. 473 43:16 10 (cited 30 Nov 2010): http://www.hesonline.org.uk/Ease/servlet/ ContentServer?siteID $=1937 \&$ categoryID $=537$.

13 Blenkharn JI, Odd C. Sharps injuries in healthcare waste handlers. Ann Occup Hyg 2008; 52(4): 281.

14 Greene ES, Berry AJ, Arnold WP, Jagger J. Percutaneous injuries in anesthesia personnel. Anesth Analg 1996; 83(2): 273.

15 Berguer R, Heller PJ. Preventing sharps injuries in the operating room. J Am Coll Surg 2004; 199(3): 462-467. 
16 Stringer B, Haines T, Goldsmith $\mathrm{CH}$, Blythe J, Berguer R, Andersen $\mathrm{J}$ et al. Hands-free technique in the operating room: reduction in body fluid exposure and the value of a training video. Public Health Rep 2009; 124(Suppl 1): 169-179.

17 Makary MA, Pronovost PJ, Weiss ES, Millman EA, Chang D, Baker SP et al. Sharpless surgery: a prospective study of the feasibility of performing operations using non-sharp techniques in an urban, university-based surgical practice. World J Surg 2006; 30(7): 1224-1229.

18 Centers for Disease Control and Prevention. Evaluation of blunt suture needles in preventing percutaneous injuries among health-care workers during gynecologic surgical procedures-New York City, March 1993-June 1994. JAMA 1997; 277(6): 451-452.

19 Bhatia SS. Ocular surface sealants and adhesives. Ocul Surf 2006; 4(3): 146-154.

20 Everts PAM, Knape JTA, Weibrich G, Schönberger JPAM, Hoffmann J, Overdevest EP et al. Platelet-rich plasma and platelet gel: a review. J Extra Corpor Technol 2006; 38(2): 174-187.
21 Panda A, Kumar S, Kumar A, Bansal R, Bhartiya S. Fibrin glue in ophthalmology. Indian J Ophthalmol 2009; 57(5): 371-379.

22 Thomas W, Murray J. The incidence and reporting rates of needle-stick injury amongst UK surgeons. Ann R Coll Surg Engl 2009; 91(1): 12-17.

23 Leliopoulou C, Waterman H, Chakrabarty S. Nurses failure to appreciate the risks of infection due to needle stick accidents: a hospital based survey. J Hosp Infect 1999; 42(1): 53-59.

24 Elmiyeh B, Whitaker IS, James MJ, Chahal CAA, Galea A, Alshafi K. Needle-stick injuries in the National Health Service: a culture of silence. J R Soc Med 2004; 97(7): 326-327.

25 Makary MA, Al-Attar A, Holzmueller CG, Sexton JB, Syin D, Gilson MM et al. Needlestick injuries among surgeons in training. $N$ Engl J Med 2007; 356(26): 2693-2699.

26 Sharma GK, Gilson MM, Nathan H, Makary MA. Needlestick injuries among medical students: incidence and implications. Acad Med 2009; 84(12): 1815-1821. 